Noname manuscript No.

(will be inserted by the editor)

\title{
On Modern Clause-Learning Satisfiability Solvers
}

\author{
Knot Pipatsrisawat · Adnan Darwiche
}

the date of receipt and acceptance should be inserted later

Keywords Satisfiability, Satisfiability solver, Clause learning, Phase selection heuristic

Abstract In this paper, we present a perspective on modern clause-learning SAT solvers that highlights the roles of, and the interactions between, decision making and clause learning in these solvers. We discuss two limitations of these solvers from this perspective and discuss techniques for dealing with them. We show empirically that the proposed techniques significantly improve state-of-the-art solvers.

\section{Introduction}

Recent progress in industrial SAT solvers allows many real-world problems to be solved in a reasonable amount of time. As a result, modern SAT solvers are being used by many researchers across many disciplines. The type of solvers that is most effective on many classes of problems stemming from real-world applications is known as clauselearning SAT solvers. These solvers are descendants of the pioneer work by Davis et al [7], which gave rise to the DPLL algorithm. Since this early work, many techniques and refinements have transformed the DPLL algorithm into modern clause-learning SAT solvers such as GRASP [22], Chaff [23], MiniSat [10], Rsat [27], and PicoSat [5].

Many models of modern SAT solvers exist in the literature (for example, see [23,29, $4,24,15]$ ). Each proposed model serves as a framework for understanding and reasoning about these solvers from different perspectives. In this work, we propose a simple model of modern clause-learning SAT solvers. We intend to make this model simple enough to allow a non-expert to understand how a modern SAT solver works and hope that, at the same time, it will provide many insights for SAT researchers.

Conventionally, clause-learning solvers are either viewed as systematic search engines or resolution engines. In this work, we take a middle approach, which emphasizes the importance of both the search and resolution components. As we describe how these two components work and interact, we will also try to justify some of the choices in modern SAT solvers, which have been taken for granted by the SAT community.

Computer Science Department, University of California, Los Angeles, 405 Hilgard Ave, Los Angeles, CA 90024, USA 
The presented model also reveals some limitations of the existing search and resolution components. In one case, we found that the choice of clauses learned by modern SAT solvers is too limited (according to the proposed measure) and that a broader class of clauses may be considered. In the other case, we found that the interaction between the search and resolution components could cause great inefficiency on problems with sub-problem structure. We discuss two techniques for dealing with these problems. One technique allows the solvers to learn shorter clauses and to backtrack further through a new class of conflict clauses. The other is a simple phase selection heuristic that can be viewed as a way of caching partial solutions without much overhead. We evaluate the effectiveness of both proposed techniques on industrial SAT problems and show that they significantly improve state-of-the-art solvers.

The rest of this paper is organized as follows. In the next section, we present a model of modern clause-learning SAT solvers. The presentation is guided by a concrete running example and followed by some observations about these solvers. Then, we discuss some limitations of existing solvers from the perspective of the presented model and propose two techniques for dealing with them in Sections 3 and 4 . Finally, we conclude in Section 5.

\section{A Simple Model of Modern SAT Solvers}

Modern SAT solvers work by repeatedly making decisions and using unit resolution to derive implications. This process is interrupted as soon as a solution or a conflict is found. If a solution is found, the problem is satisfiable and the solver terminates. If a conflict is found, there are two cases to consider. First, if the conflict occurs in the absence of any decisions, the problem is unsatisfiable and the solver can terminate. Otherwise, the solver derives a conflict clause, rolls back some decisions (backtracks), adds the clause to the knowledge base, and continues making decisions again. A pseudocode of modern SAT solver is shown in Algorithm $1 .^{1}$

From this description, one can view each solver as consisting of two equally important components: a decision-making engine and a clause-learning engine. The decisionmaking engine, whose goal is to find a satisfying assignment, is responsible for making decisions (assignments) and deriving implications from the decisions. The clauselearning engine, whose goal is to strengthen unit resolution by learning clauses, is responsible for deriving learned clauses and backtracking. In Algorithm 1, it is clear that the first line of code is carried out by the decision-making engine, while the forth line is performed by the clause-learning engine.

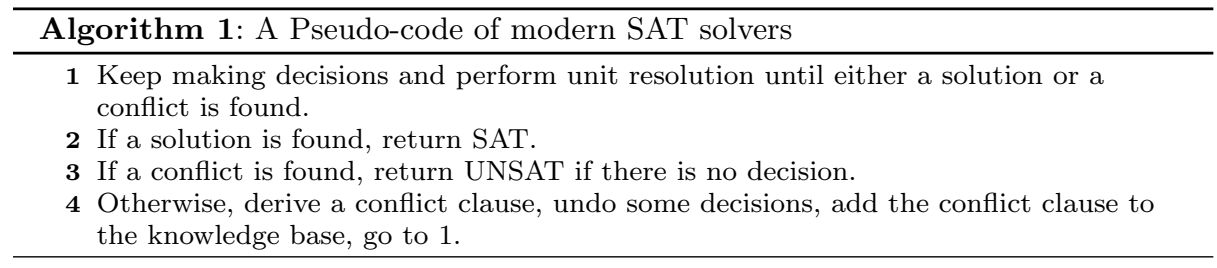

1 We intentionally left out some details of the algorithm to emphasize its high-level structure. We will present more details of important components in the following discussion. 


\subsection{An Example Trace of a Modern SAT Solver}

Let us now show an example execution of a typical modern SAT solver. Consider the following CNF:

$$
\begin{aligned}
\Delta= & (\neg a \vee \neg b \vee \neg y \vee h), \\
& (\neg a \vee \neg b \vee \neg y \vee \neg h), \\
& (\neg a \vee b \vee \neg y \vee h), \\
& (\neg a \vee b \vee \neg y \vee \neg h), \\
& (\neg c \vee \neg z \vee y), \\
& (\neg d \vee e), \\
& (\neg d \vee \neg e \vee z), \\
& (\neg x \vee f \vee g), \\
& (\neg x \vee f \vee \neg g), \\
& (\neg x \vee \neg f \vee g), \\
& (\neg x \vee \neg f \vee \neg g) .
\end{aligned}
$$

The solver begins by making decisions until either a solution or a conflict is found. Let's assume that, in this example, the variables are chosen as decisions in alphabetical order and that they are set to true. Therefore, the first three decisions will be $a, b, c$, none of which allows unit resolution to derive any unit implications. At this point, the simplified CNF formula under the current assignments is

$$
\begin{aligned}
\Delta^{\prime}= & (\neg y \vee h), \\
& (\neg y \vee \neg h), \\
& (\neg z \vee y), \\
& (\neg d \vee e), \\
& (\neg d \vee \neg e \vee z), \\
& (\neg x \vee f \vee g), \\
& (\neg x \vee f \vee \neg g), \\
& (\neg x \vee \neg f \vee g), \\
& (\neg x \vee \neg f \vee \neg g) .
\end{aligned}
$$

As soon as the next decision, $d$, is made, unit resolution will be able to derive $e, z, y, h, \neg h$ as implications. Clearly, there is a conflict; both $h$ and $\neg h$ are implied. Table 1 shows the decisions and implications at this point in time. As in conventional formulation, we associate each decision with a positive integer which indicates its level.

\begin{tabular}{|l|c|c|c|c|c|}
\hline Levels & 0 & 1 & 2 & 3 & 4 \\
\hline Decisions & - & $a$ & $b$ & $c$ & $d$ \\
\hline Unit implications & - & - & - & - & $e, z, y, h, \neg h$ \\
\hline
\end{tabular}

Table 1 The state of the solver at the first conflict. 
Before we talk about clause learning and backtracking for this conflict, let us first pay attention to the assignments which are logically implied right before the conflict (after setting $c=$ true at level 3). Whenever a conflict is discovered, it simply means that the current formula, together with all but the last decisions, implies the negation of the last decision, yet unit resolution did not see this implication. In this case, $\Delta$, together with $a, b, c$, implies $\neg d$. In general, more than one implication might be missed by unit resolution at the time of conflict. In this example, four implications are missed after $c$ is set to true: $\neg x, \neg y, \neg z$, and $\neg d$. Not every implication is missed in the same way, however. In particular, for some implications, even though unit resolution could not derive them, they could be derived by unit refutation (i.e., unit resolution will detect a conflict if the negation of the missed implication is asserted). We say that these literals are weakly missed implications. Other missed implications that cannot be derived this way are called strongly missed implications. The notions of weakly missed implications and strongly missed implications are dependent on the state (level) of the solver. Moreover, it is not very meaningful to talk about these missed implications when there is a conflict (i.e., the knowledge base and the decisions are already inconsistent). Hence, in future discussions, whenever we refer to missed implications in the presence of a conflict, we mean those missed implications that exist before the last decision that leads to the conflict.

\begin{tabular}{|l|c|c|c|c|}
\hline Levels & 0 & 1 & 2 & 3 \\
\hline Decisions & - & $a$ & $b$ & $c$ \\
\hline Strongly missed implications & $\neg x$ & $\neg x, \neg y$ & $\neg x$ & $\neg x$ \\
\hline Weakly missed implications & - & - & $\neg y$ & $\neg y, \neg z, \neg d$ \\
\hline
\end{tabular}

Table 2 Missed implications.

The strongly missed implications and weakly missed implications in this example are shown in Table 2 . After the decision at level 3 , we have $\neg y, \neg z, \neg d$ as weakly missed implications and $\neg x$ as a strongly missed implication. ${ }^{2}$ Since every conflict indicates a missed implication, one can view each conflict as an opportunity to empower unit resolution to see some of these missed implications. To achieve this, the solver will learn what is called an asserting clause, which can be defined as follows. An asserting clause is simply a clause of the form $\alpha \Rightarrow \ell$ such that $\alpha$ is a subset of the literals implied by unit resolution before the last decision and $\ell$ is falsified at the level of conflict. Moreover, asserting $\alpha \wedge \neg \ell$ in the current knowledge base (of original and learned clauses) results in a conflict after applying unit resolution. These conditions automatically imply that $\ell$, which is called the asserted literal of the clause, must be a weakly missed implication before the last decision was made. The maximum level at which any literal of $\alpha$ is implied is referred to as the assertion level of the clause. Interestingly, the assertion level is also the level at which $\ell$ became weakly missed. Clearly, every asserting clause becomes unit at its assertion level, thus directly allowing unit resolution to see the weakly missed implication.

The process of clause learning involves analyzing the trace of unit resolution, which will allow the solver to identify a subset of the weakly missed implications and their associated asserting clauses. The solver then selects to learn the asserting clause with

2 It is possible for a strongly missed implication to become a weakly missed implication like $\neg y$ in this case. We will discuss this phenomenon in more details later. 
the earliest assertion level. In the above example, the asserting clause $C=(a \wedge b) \Rightarrow$ $\neg y=(\neg a \vee \neg b \vee \neg y)$, which targets the weakly missed implication $\neg y$, will be learned. ${ }^{3}$ The solver will then backtrack to the assertion level (level 2) and add this clause to the knowledge base. Table 3 shows the state of the solver right after $C$ is added and unit resolution is applied. Since this results in no conflict, the decision-making engine will continue making decisions. Next, we make a few observations about modern SAT solvers in the context of the above example, allowing us to more formally justify the claims we made about the behavior of modern SAT solvers.

\begin{tabular}{|l|c|c|c|}
\hline Levels & 0 & 1 & 2 \\
\hline Decisions & - & $a$ & $b$ \\
\hline Unit implications & - & - & $\neg y$ \\
\hline \hline Strongly missed implications & $\neg x$ & $\neg x, \neg y$ & - \\
\hline Weakly missed implications & - & - & - \\
\hline
\end{tabular}

Table 3 The state of the solver after adding $(\neg a \vee \neg b \vee \neg y)$.

\subsubsection{Identifying Weakly Missed Implications}

Whenever the solver discovers a conflict, it produces a proof that the knowledge base is unsatisfiable under the current decision sequence. Such a proof is called a unit refutation proof, which is simply a derivation of the empty clause (i.e., the truth constant false) from the current knowledge base and the decisions using unit resolution. From such a proof, a number of weakly missed implications can be identified and asserting clauses can be derived.

A unit refutation proof can be visualized with a graph which shows how each unit implication is derived (leading to the derivation of the empty clause). This graph is also known as implication graph $[22,23]$. Figure 1 shows such a graph for the conflict in the above example. In this graph, the parents of each node $\ell$ are literals which were used by unit resolution to resolve with a clause to produce a unit clause that contains $\ell$ (decision literals do not have any parent). The clause in which a literal becomes unit is called the reason of that literal. For example, $(\neg d \vee \neg e \vee z)$ is the reason of $z$ at the time of the conflict in the above example.

If $D$ is the node in the implication graph which corresponds to the decision that leads to the conflict, any node that dominates the false node with respect to $D$ is known as a unique implication point (UIP) $[22] .{ }^{4}$ In the graph in Figure 1, y,z, and $d$ are the UIPs. Interestingly, the negations of the UIPs are guaranteed to be weakly missed implications before the conflict. In some conflicts, there may be multiple unit refutation proofs. In such situations, different proofs used by unit resolution may lead to different subsets of the weakly missed implications identified by the solver. The choice of refutation proof used by the solvers is automatically controlled by the implementation of unit resolution.

In practice, modern SAT solvers learn the asserting clause associated with the UIP that is closest to the false node of the implication graph (also known as first

3 We will discuss the details of the derivation in a later section.

4 Node $x$ dominates $y$ with respect to $z$ iff every (undirected) path from $y$ to $z$ has to go through $x$. 


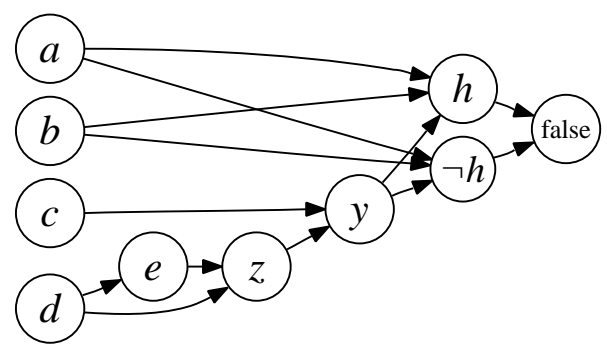

Fig. 1 A graph showing a derivation of a conflict.

UIP [23]). Because first UIP asserting clauses always induce the farthest backtracks [2], the learning of first UIP asserting clauses in modern SAT solvers can be viewed as a way of making sure that the implication which was (weakly) missed the earliest (in the considered implication graph) is targeted.

\subsubsection{Strongly and Weakly Missed Implications}

As more decisions are made and more clauses are added, some strongly missed implications may turn into weakly missed implications, while others stay unchanged. For instance, $\neg x$ is a strongly missed implication that has never become a weakly missed implication in the above example (see Table 2 ). Literals $\neg z$ and $\neg d$ became weakly missed implications from the first moment they were missed. Literal $\neg y$, on the other hand, was originally a strongly missed implication at level 1 but eventually turned into a weakly missed implication after the second decision was made. In general, there could be a potentially significant difference between the point at which an implication is first missed and the point at which it becomes a weakly missed implication (if it does at all).

\subsubsection{Limitations of Learned Clauses}

At each conflict, modern SAT solvers learn an asserting clause that helps unit resolution realize one of the weakly missed implications at an earlier level. This kind of clauses actually satisfy a very specific property. In particular, not only are these clauses entailed by the knowledge base, but their entailment can actually be proven by unit resolution. In other words, for each asserting clause $C$ learned by modern solvers, adding $\neg C$ to the knowledge base (which contains the original and previously learned clauses) will result in a conflict (using unit resolution) [14].

Interestingly, for every weakly missed implication $\ell$, we can always find an asserting clause with $\ell$ as the asserted literal. However, no clause with such a property exist for any strongly missed implication, because if asserting $\neg C$ results in a conflict (using unit resolution), the asserted literal must be a weakly missed implication by definition. An implication of this observation is that modern SAT solvers cannot directly empower unit resolution with respect to any strongly missed implications in a single conflict (using a single asserting clause). To enable unit resolution to see a strongly missed 
implication, multiple clauses need to be learned from multiple conflicts. ${ }^{5}$ For instance, at the conflict of the above example, modern SAT solvers would not be able to add a single asserting clause which allows $\neg x$ to be implied.

\subsubsection{Decision Sequence May be Repeated}

In the above example, after the clause $(\neg a \vee \neg b \vee \neg y)$ is added and the solver resumes the search, it is possible for the solver to repeat the same decision sequence ( $c=$ true, $d=$ true), after which the solver will run into another conflict. Table 4 shows the decisions and implications after the same decision sequence has been repeated.

This illustrates the fact that modern SAT solvers have substantially deviated from the traditional systematic (depth-first) search algorithm. As a result, we believe it is more accurate to describe the search performed in modern SAT solvers as a greedy search, which tries to find a solution as quickly as possible, rather than a systematic search in the space of variable assignments. Of course, the completeness of the algorithm is not compromised thanks to unit resolution and clause learning.

\begin{tabular}{|l|c|c|c|c|c|}
\hline Levels & 0 & 1 & 2 & 3 & 4 \\
\hline Decisions & - & $a$ & $b$ & $c$ & $d$ \\
\hline Unit implications & - & - & $\neg y$ & $\neg z$ & $e, \neg e$ \\
\hline \hline Strongly missed implications & $\neg x$ & $\neg x, \neg y$ & $\neg x$ & $\neg x$ & \\
\hline Weakly missed implications & - & - & - & $\neg d$ & \\
\hline
\end{tabular}

Table 4 The state of the solver after repeating the decision sequence and discovering the conflict.

\subsection{Related Work}

The authors of [19] used the notation failed literal to refer to the negation of a weakly missed implication. The idea of identifying missed implications has been studied in the past. For example, in [20], a technique called unit propagations of second level was studied in the context of random formulas. In [18], the author formalized various strategies involving different usages of unit resolution in order to detect missed implications. Preprocessing SAT formulas (see $[3,21,31,11]$, for examples) can also be viewed as a one-time attempt to use more expensive inference rules to identify implications that cannot be detected by unit resolution.

This concludes the discussion of our model of modern SAT solvers. In the following sections, we discuss two limitations observed from the model described and propose improvements, which are implemented through the decision-making and clause-learning engines of modern SAT solvers.

\footnotetext{
5 After enough asserting clauses have been added, the strongly missed implication will turn into a weakly missed implication, which can then be fixed by a single asserting clause.
} 


\section{Empowering Unit Resolution}

In this section, we discuss a limitation of the current clause-learning approach utilized by virtually all modern clause-learning SAT solvers and propose a way of improving it. Current clause-learning algorithms are based on learning an asserting clause upon each conflict. We hypothesize that one property, called empowerment (to be defined), is crucial for solving unsatisfiable problems. Interestingly, our investigation reveals that some non-asserting clauses also satisfy this property. Therefore, considering only asserting clauses limits the possibility of learning clauses that may be equally useful, yet are better than asserting clauses in terms of metrics such as clause size and backtrack distance.

Later in this section, we propose a new clause-learning algorithm, which considers a broader class of conflict clauses that satisfy the empowerment property (in a sense to be made precise later). As a result, the new learning algorithm may sometimes yield shorter clauses and longer backtracks, allowing the clause-learning engine to derive stronger clauses and target implications that are missed earlier. Finally, we show empirically that the proposed technique significantly improves the performance of a modern SAT solver on unsatisfiable problems. We begin our discussion by introducing the notion of empowerment, which lies at the heart of the observed limitation and the proposed improvement.

\subsection{Empowerment}

Given a CNF $\Delta$ and a clause $C$ implied by $\Delta, C$ is empowering with respect to $\Delta$ if $C$ allows unit resolution to derive, under some assignment, a new implication that would not be derivable without the clause. ${ }^{6}$ In other words, $C$ enables unit resolution to see an implication that it could not see before. For example, consider the CNF $\Delta=(a \vee b) \wedge(\neg a \vee c) \wedge(b \vee \neg c \vee d)$, and the clause $(b \vee d)$ which is implied by the $\mathrm{CNF}$. Adding $\neg d$ to $\Delta$ does not allow unit resolution to derive $b$ even though $b$ is implied by $\Delta \wedge \neg d$. Yet, this derivation becomes possible once we add the clause $(b \vee d)$ to $\Delta$. Hence, the clause is empowering with respect to $\Delta$. In contrast, $(b \vee c)$ is not an empowering clause with respect to the same CNF, because asserting $\neg b$ (resp. $\neg c$ ) allows unit resolution to derive $c$ (resp. $b$ ) from $\Delta$ even without the clause. We refer to any literal in an empowering clause that corresponds to the new implication as an empowering literal.

Every asserting clause learned by modern SAT solvers is empowering with respect to the knowledge base at the time of learning. Nevertheless, asserting clauses are not the only type of clauses that are empowering. For example, consider

$$
\begin{aligned}
\Delta= & (\neg a \vee \neg b \vee \neg c \vee d), \\
& (\neg c \vee e), \\
& (\neg d \vee \neg e \vee f), \\
& (\neg e \vee \neg f) .
\end{aligned}
$$

Assume that the solver makes the decisions $a, b, c$ in this order. At this point, $d, e, f, \neg f$ are implied and there is a conflict. The current state of the solver is shown in Table 5.

${ }^{6}$ For simplicy, we use the term empowering instead of 1-empowering originally used in [28]. 
An asserting clause for this conflict is $(\neg a \vee \neg b \vee c)$. While this clause is empowering with respect to $\Delta$, the clauses $(\neg d \vee \neg e)$ and $(\neg c \vee \neg d)$, which are also falsified by the current assigment, are empowering with respect to $\Delta$ as well. Note that these clauses are not asserting clauses with respect to this conflict, because $c, d, e$ are all assigned at the level of the conflict. This observation suggests that non-asserting clauses could also be learned for the purpose of empowering unit resolution. This leads to the definition of a new class of clauses which we introduce next.

\begin{tabular}{|l|c|c|c|c|}
\hline Levels & 0 & 1 & 2 & 3 \\
\hline Decisions & - & $a$ & $b$ & $c$ \\
\hline Unit implications & - & - & - & $d, e, f, \neg f$ \\
\hline \hline Strongly missed implications & - & - & - & \\
\hline Weakly missed implications & - & - & $\neg c$ & \\
\hline
\end{tabular}

Table 5 The state of the solver at a conflict.

\subsection{Bi-Asserting Clauses}

If the main goal of the clause-learning engine is to empower unit resolution, then it seems too restrictive to consider only the asserting subset of empowering clauses. In this section, we discuss a different class of clauses that may be learned at each conflict. By broadening the set of clauses that the solvers consider for learning, the solvers will have more opportunities for learning a useful clause.

The new class of clauses also targets missed implications like asserting clauses do. However, instead of allowing unit resolution to see a missed unit implication, they will allow unit resolution to see weakly missed binary implications. A binary clause is a weakly missed binary implication if the clause or any of its literals cannot be derived by unit resolution, yet asserting its negation results in a conflict (using unit resolution). For example, $(\neg b \vee \neg c)$ is a weakly missed binary implication after $a$ is set to true in the above example.

The new class of clauses is called bi-asserting clauses [28]. A bi-asserting clause is defined in a similar way as an asserting clause. An asserting clause contains exactly one literal falsified at the level of conflict. A bi-asserting clause, on the other hand, contains exactly two literals falsified at the level of conflict. More formally, $C=\alpha \Rightarrow\left(\ell_{1} \vee \ell_{2}\right)$ is a bi-asserting clause iff (i) $\alpha$ is a subset of the literals implied by unit resolution before the last decision, (ii) $\ell_{1}, \ell_{2}$ are falsified at the conflict level, and (iii) asserting $\neg C$ in the current knowledge base leads to a conflict detectable by unit resolution. The assertion level of a bi-asserting clause is the largest level that any literal in $\alpha$ was implied. In the previous example, both $(\neg d \vee \neg e)$ and $(\neg c \vee \neg d)$ are bi-asserting clauses (their $\alpha$ 's are true). Moreover, these binary clauses are weakly missed binary implications at level 0 . As mentioned earlier, these two bi-asserting clauses are also empowering.

Deriving a bi-asserting clause is not any harder than deriving an asserting clause. The standard algorithm for deriving asserting clauses can be easily modified to consider bi-asserting clauses. Algorithm 2 is a pseudo-code of the asserting clause derivation algorithm (based on the one described in [29]). This algorithm works by resolving clauses that become falsified and unit during unit resolution together (in reverse order) 
until the resolvent contains only 1 literal falsified at the last level, at which the conflict takes place. In this algorithm, we use the notation litsAtConflictLevel $(C)$ to refer to the set of literals of $C$ that are falsified at the level of conflict.

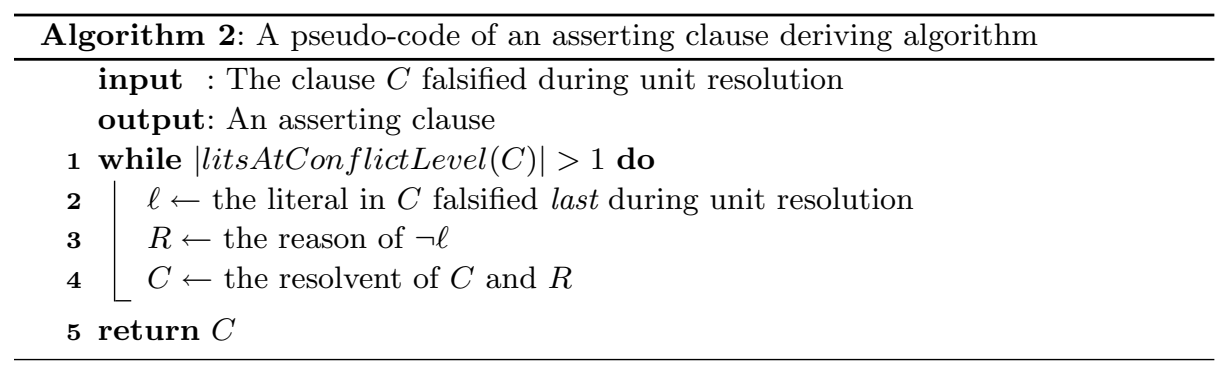

To derive a bi-asserting clause, we only need to modify the test condition of the while loop to continue as long as more than two literals in $C$ are falsified at the current level (e.g. $\mid$ litsAtConflictLevel $(C) \mid>2$ ). We will later show empirically that bi-asserting clauses tend to be shorter and induce farther backtracks than asserting clauses, thus establishing their benefits.

\subsection{Empowering Bi-Asserting Clauses}

Unlike asserting clauses which are always empowering, bi-asserting clauses may not be empowering with respect to the knowledge base at the time of learning. If the goal of the clause-learning engine is to allow unit resolution to see new implications, then it does not make sense to learn a non-empowering clause, because they can never generate any new implication. Therefore, in our discussion of bi-asserting clauses, we will only focus on those bi-asserting clauses that are also empowering.

A problem that we now face is how to determine whether a bi-asserting clause is empowering. In general, checking whether a clause is empowering with respect to CNF $\Delta$ can be done by asserting, for each literal in the clause, the negations of the other literals and checking whether unit resolution can derive the literal. The time complexity of this test is linear in the size of the clause and in the size of the knowledge base. In practice, however, this test would incur too much overhead. Therefore, instead of insisting on learning bi-asserting clauses which are empowering with respect to all clauses in the knowledge base, we will only require them to be locally empowering-that is, empowering with respect to the clauses used in their derivations. This reduces the overhead significantly. We will later show, through empirical experiments, that, in most cases, clauses which are locally empowering actually turn out to be empowering with respect to the whole knowledge base as well.

Our approach for ensuring local empowerment makes use of the notion of merge resolution [1]. A merge resolution is simply a resolution between two clauses which share at least one common literal. A common literal of a merge resolution is referred to as a merged literal. For example, the resolution between $(a \vee b \vee c)$ and $(\neg a \vee b \vee d)$ is a merge resolution and $b$ is a merged literal. On the other hand, the resolution between $(a \vee b \vee c)$ and $(\neg a \vee d \vee e)$ is not a merge resolution.

It turns out that if, during the derivation of a bi-asserting clause, there is a merge resolution step, then the resulting bi-asserting clause will be empowering with respect to the clauses in the current knowledge base that are used in the derivation (i.e., the 
initial empty clause and clauses $R$ in Algorithm 2). Moreover, the merged literals, which could have been implied at any level, that appear in the resulting clause (there has to be at least one such literal) will all be empowering literals. We formalize and prove this result in the Appendix (Proposition 1). This result gives us an easy way to detect whether a bi-asserting clause (or any resolvent) derived this way will be locally empowering or not. All we need to do is to look for a merge resolution step; whenever one is performed, we are ensured that the output will be locally empowering.

The purpose of learning a bi-asserting clause is to allow unit resolution to see a weakly missed binary implication. However, this will be more useful when the weakly missed binary implication later materializes into a new unit implication. Therefore, it makes more sense, when we try to learn an empowering bi-asserting clause, to insist that at least one of the literals of the weakly missed binary implication of the biasserting clause be an empowering literal of the clause. This amounts to making sure that at least a literal at the level of conflict is a merged literal during the derivation of the clause. Algorithm 3 shows the aforemention algorithm for deriving (locally) empowering bi-asserting clauses. The variable curLevelMerge becomes true whenever a literal falsified at the conflict level has been merged. According to the conditions of the while loop, this algorithm will derive a bi-asserting clause only when some literal at the conflict level has been merged in the derivation. If no such clause is found, the algorithm automatically falls back to the first-UIP asserting clause, which can always be derived at every conflict.

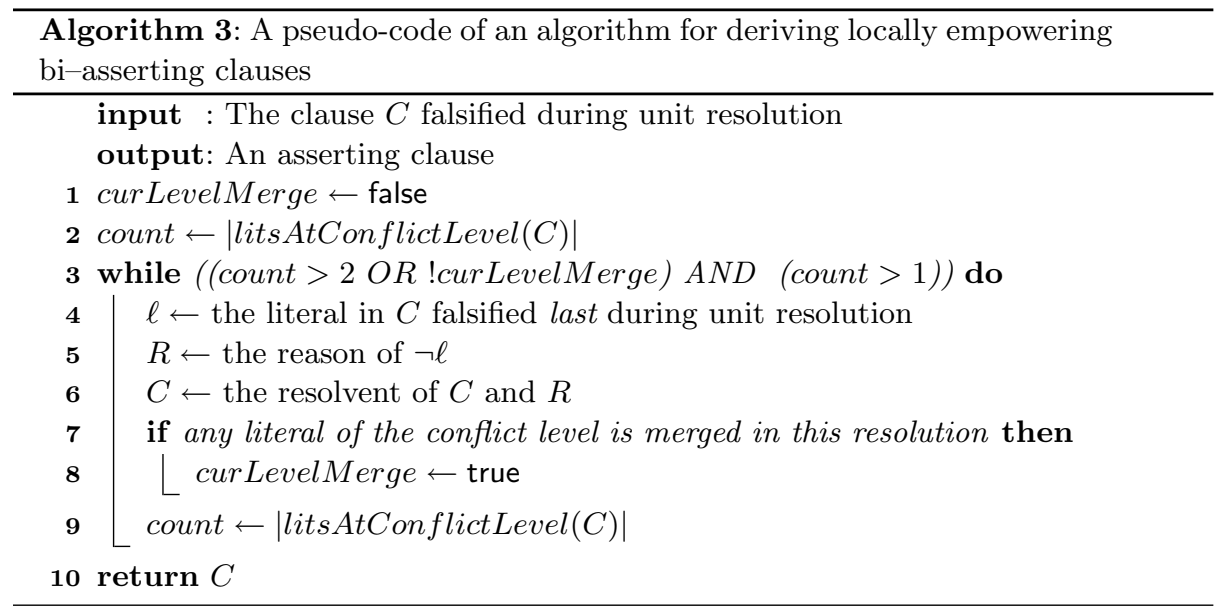

We define the assertion level of a bi-asserting clause to be the second-highest level at which any literal in the clause is falsified. Whenever a bi-asserting clause is learned, no unit implication will be produced at the assertion level (the bi-asserting clause will contain two free literals). In this work, we do not impose any special condition on the decision making after a bi-asserting clause is learned (i.e., the default variable ordering heuristic, VSIDS [23], is always used). ${ }^{7}$

\footnotetext{
7 We experimented with different decision heuristics for forcing bi-asserting clauses to become unit after learning, but found no significant improvement. Therefore, we decided to always use the default heuristic to simplify the implementation.
} 


\subsection{Experimental Results}

In this section, we present experimental results that show the benefits of learning empowering bi-asserting clauses. We modified Rsat [27] (without the preprocessor), the winner of the industrial category of the SAT'07 competition (SAT+UNSAT and UNSAT specialties), to detect any occurrence of locally empowering bi-asserting clauses during conflict clause derivation (as described in the previous section). If found, the bi-asserting clause is learned instead of the asserting clause otherwise learned. We call this version of the solver Rsat+.

Although empowerment mentioned here is only with respect to the clauses used in the derivation, in practice, it usually results in empowerment with respect to the whole formula. We used the procedure described in the previous section to measure the percentage of bi-asserting clauses that are actually empowering with respect to the whole formula when learned. We found that on $95 \%$ of the problems more than $80 \%$ of the bi-asserting clauses derived this way are empowering with respect to the whole formula. This shows that our algorithm approximates empowerment well in practice. To evaluate the importance of empowerment, we also tested the version of Rsat that learns bi-asserting clauses regardless of their empowerment (if one is found). We call this version Rsat-.

We experimented with nearly 1,000 problems from previous SAT/SAT-Race competitions and contemporary benchmark libraries. ${ }^{8}$ Each solver is given 1,800 seconds per problem on a $3.8 \mathrm{GHz}$ computer with $4 \mathrm{~GB}$ of RAM.

On satisfiable problems, the behaviors of Rsat and Rsat+ are comparable. Rsat solved 338 problems, while Rsat+ solved 336 problems. Moreover, Rsat+ used about $19 \%$ more time on the problems that both solvers could solve. This result shows that learning empowering bi-asserting clauses slightly worsens the performance on satisfiable problems. This result is expected as bi-asserting clauses are not as aggressive as asserting clauses in generating new implications, which help the solver discover a solution more quickly. Rsat-, however, solved only 295 satisfiable problems, showing that learning non-empowering bi-asserting clauses does worsen the performance.

On unsatisfiable problems, we observed more difference in performance between these solvers. Table 6 highlights the results on unsatisfiable problems. For each family, we report the total number of problems, the number of problems solved by each solver, and the total running time of each solver on the problems that it solved. The result of the best solver in each family is highlighted (based on number solved, ties broken by running time).

According to the result on unsatisfiable problems, Rsat- performed worse than even the original version of Rsat on most of the families. Overall, Rsat- solved 48 fewer problems than Rsat. This clearly suggests that learning just any bi-asserting clause is not a good idea for either satisfiable or unsatisfiable problems. This is because these biasserting clauses are not necessarily capable of generating any new implications. So, in the long run, the non-empowering clauses only contribute to the overhead of unit resolution no matter how short they are. Now, comparing Rsat against Rsat+ shows that learning empowering bi-asserting clauses allowed Rsat+ to solve some unsatisfiable problems in the vliw_unsat families (last 3 families in Table 6). These problems

\footnotetext{
8 http://www.satcompetition.org/2007, http://fmv.jku.at/sat-race-2006, http://www.research.ibm.com/haifa/projects/verification/RB_Homepage/fvbenchmarks.html, http://miroslav-velev.com/sat_benchmarks.html.
} 


\begin{tabular}{|l|r||r|r|r|r|r|r|}
\hline Family & \multicolumn{1}{|c|}{ Total } & \multicolumn{3}{|c|}{ \# solved } & \multicolumn{3}{|c|}{ Running time (s) } \\
\cline { 3 - 8 } & & Rsat & Rsat- & Rsat+ & Rsat & Rsat- & Rsat+ \\
\hline \hline dlx_iq_unsat_1 & 32 & 10 & 0 & $\mathbf{1 6}$ & 12812.40 & 0 & 17537.43 \\
\hline engine & 10 & 7 & 5 & 7 & 1288.35 & 980.51 & $\mathbf{9 0 2 . 5 2}$ \\
\hline fvp_unsat_1 & 4 & 4 & 4 & 4 & 39.63 & 196.45 & $\mathbf{2 1 . 5 4}$ \\
\hline fvp_unsat_2 & 22 & 21 & 21 & 21 & 1842.56 & 1355.41 & $\mathbf{4 2 8 . 3 6}$ \\
\hline IBM & $165 *$ & 164 & 164 & $\mathbf{1 6 5}$ & 1320.17 & 4550.83 & 3334.69 \\
\hline liveness_unsat_1 & 12 & 4 & 3 & 4 & 1329.57 & 209.01 & $\mathbf{6 4 4 . 6 0}$ \\
\hline liveness_unsat_2 & 9 & 3 & 3 & 3 & 92.51 & 182.93 & $\mathbf{6 5 . 8 8}$ \\
\hline narain 2005 & 4 & 2 & 2 & 2 & 558.57 & 827.6 & $\mathbf{4 5 2 . 5 7}$ \\
\hline pipe_ooo & 29 & 12 & 11 & $\mathbf{1 4}$ & 4559.63 & 2159.99 & 3738.83 \\
\hline pipe_unsat_1.0 & 13 & 7 & 7 & $\mathbf{1 0}$ & 2192.66 & 1573.73 & 3044.54 \\
\hline pipe_unsat_1.1 & 14 & 7 & 7 & $\mathbf{1 0}$ & 1243.38 & 2446.93 & 2620.46 \\
\hline SAT-Race'06 Final & 57 & 45 & 33 & $\mathbf{5 1}$ & 13518.37 & 9340.93 & 12562.65 \\
\hline SAT-Race'06 Q1 & 31 & 28 & 28 & $\mathbf{3 0}$ & 2139.67 & 8804.411 & 2365.57 \\
\hline SAT-Race'06 Q2 & $29 *$ & 28 & 18 & $\mathbf{2 9}$ & 8588.72 & 2298.75 & 8188.95 \\
\hline SAT Comp. 07 & $61 *$ & 58 & 46 & $\mathbf{5 9}$ & 12856.12 & 12828.56 & 12795.59 \\
\hline vliw_unsat_2 & 9 & 0 & 0 & $\mathbf{3}$ & 0 & 0 & 2823.84 \\
\hline vliw_unsat_3 & 2 & 0 & 0 & $\mathbf{2}$ & 0 & 0 & 1056.40 \\
\hline vliw_unsat_4 & 4 & 0 & 0 & $\mathbf{1}$ & 0 & 0 & 641.82 \\
\hline \hline Total & 504 & 400 & 352 & $\mathbf{4 3 1}$ & 64382.38 & 47756.17 & 73226.34 \\
\hline
\end{tabular}

Table 6 Performance of Rsat with different learning schemes. * These numbers are not the actual number of unsatisfiable problems in these families, because not every problem could be solved and the information on the number of unsatisfiable problems in these families is not available. Therefore, we only report total instance count based only on results from our experiment.

\begin{tabular}{|l|c|c|}
\hline \multirow{2}{*}{ Family } & \multicolumn{2}{|c|}{ Avg. ratio } \\
\cline { 2 - 3 } & bt. depth & cls. size \\
\hline \hline difp & 6.86 & 0.33 \\
\hline dlx iq unsat 1 & 4.70 & 0.49 \\
\hline fpga & 4.31 & 0.64 \\
\hline fvp unsat 1 & 4.68 & 0.53 \\
\hline IBM & 4.40 & 0.43 \\
\hline pipe ooo & 6.37 & 0.48 \\
\hline SAT Comp. 07 & 6.04 & 0.41 \\
\hline SAT-Race final & 5.88 & 0.42 \\
\hline vliw unsat 2 & 6.01 & 0.56 \\
\hline
\end{tabular}

Table 7 Ratios of backtrack depths and learned clause sizes from representative families (bi-asserting clause/asserting clause).

are quite difficult for state-of-the-art solvers. According to the results available elsewhere $^{9}$, MiniSat (versions 1.14 and 2.0) could not solve any of these problems, while TiniSat (with and without preprocessor) solved 4 problems (based on a comparable time-out). Our experiment shows that Rsat with bi-asserting clause learning solved 6 of these problems under 30-minute timeout. Learning bi-asserting clauses also helped Rsat solve many more problems from other families, notably dlx_iq_unsat_1, pipe_unsat, and problems from SAT/SAT-Race competitions. Overall, Rsat+ solved 31 problems more than Rsat. Moreover, for those families in which both solvers solved the same number of problems, Rsat+ consistently took less time.

9 http://users.rsise.anu.edu.au/ jinbo/tinisat/results07cp. 


\begin{tabular}{|c|c|c|c|}
\hline \multirow{2}{*}{ Version } & \multicolumn{3}{|c|}{ Number of solved problems } \\
\cline { 2 - 4 } & SAT & UNSAT & Total \\
\hline Rsat & 338 & 400 & 738 \\
Rsat+ & 336 & 431 & 767 \\
Rsat-3 & 327 & 427 & 754 \\
Rsat-4 & 323 & 429 & 752 \\
Rsat-10 & 317 & 434 & 751 \\
\hline
\end{tabular}

Table 8 Number of problems solved by Rsat with different learning algorithms.

Further investigations shed light on the effectiveness of empowering bi-asserting clauses. We use the term backtrack depth to refer to the number of levels backtracked by the solver at each conflict. We measure the average sizes of asserting and bi-asserting clauses, and the average backtrack depths induced by these clauses during the execution of Rsat+. Table 7 shows the results of this investigation. The first column reports the names of the families of problems. The second column shows the ratios of average backtrack depth induced by bi-asserting clauses over the average depth induced by asserting clauses. The third column shows the same information but with respect to the sizes of the learned clauses. On average, a bi-asserting clause induces 4-7 times larger backtrack depth than an asserting clause for the same problem. Moreover, the size of a bi-asserting clause, on average, is less than half the size of an asserting clause for the same problem. These observations support the fact that Rsat+ outperformed Rsat on unsatisfiable problems. To prove unsatisfiability, SAT solvers have to exhaust the search space and learn enough clauses to detect a conflict at the top level. Learning empowering bi-asserting clauses clearly allows the solver to learn shorter clauses and backtrack further, thus pruning the search space more and allowing the solver to discover conflicts at shallower levels faster.

In our study, we also experimented with various variations of the learning algorithm. For example, we tried allowing the solver to learn locally empowering clauses with different number of literals at the conflict level (i.e., $N$-asserting clauses, where $N>2$ ). This only amounts to changing the test count $>2$ to count $>N$ for the while loop in Algorithm 3. We experimented with $N=3$, 4, and 10 (Rsat-3, Rsat-4, Rsat-10 respectively). Table 8 shows the number of problems solved by different versions of Rsat. According to the result, we found that allowing more literals at the conflict level (more than 2) does not significantly change the behavior on unsatisfiable problems, but worsen performance on satisfiable problems noticeably (compared to Rsat+). Overall, we found that setting $N=2$ (learning bi-asserting clauses) yields the best performance.

In the next experiment, we varied the amount of bi-asserting clauses that the solver learned by introducing a threshold $M$ on the number of merge resolutions. In this experiment, the solver would learn a bi-asserting clauseat a conflict (based on Algorithm 3) only if the number of merge resolutions in its derivation is greater than or equal to $M$. Setting $M$ to different values allow us to see the impacts of learning different amounts of bi-asserting clauses. Figure 2 shows the percentage of bi-asserting clauses (vs all learned clauses) learned by different variations of Rsat in each problem. ${ }^{10}$ In this experiment, we set $M$ to be 1 (as in Rsat+), 2, 5, and 10. This figure shows that the larger $M$ gets the smaller the percentage of bi-asserting clauses learned becomes. In terms of performance, we found that setting $M=1$ yielded the best result. When $M=$ 2,5 , and 10, the solver solved 7,22, and 32 fewer problems respectively. Table 9 reports

10 For each solver, the problems are ordered by the percentages of bi-asserting clauses learned. 


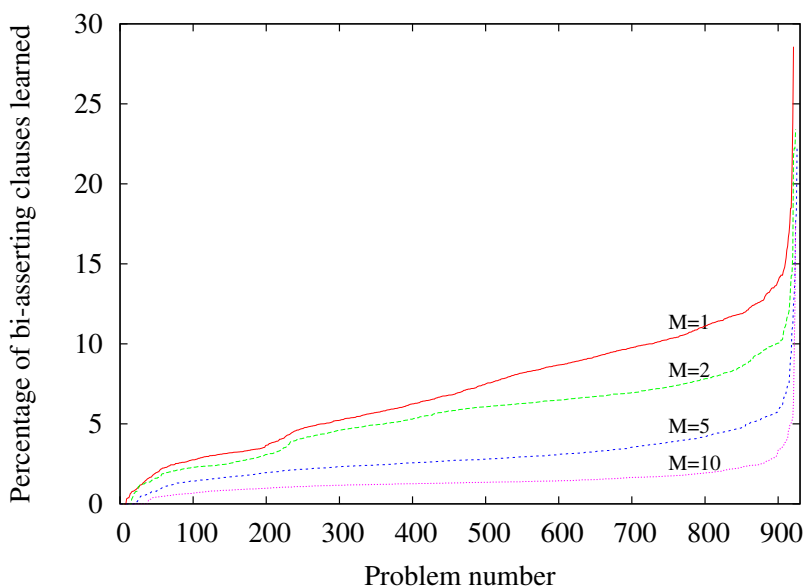

Fig. 2 Percentages of bi-asserting clauses over all learned clauses learned by Rsat with different merge thresholds.

\begin{tabular}{|c|c|c|c|}
\hline \multirow{2}{*}{ Version } & \multicolumn{3}{|c|}{ Number of solved problems } \\
\cline { 2 - 4 } & SAT & UNSAT & Total \\
\hline Rsat & 338 & 400 & 738 \\
Rsat+ $(M=1)$ & 336 & 431 & 767 \\
Rsat+ $M=2)$ & 345 & 415 & 760 \\
Rsat+ $(M=5)$ & 338 & 407 & 745 \\
Rsat+ $(M=10)$ & 335 & 400 & 735 \\
\hline
\end{tabular}

Table 9 Number of problems solved by Rsat+ with different merge thresholds.

more detailed results of this experiment. These results show that as the threshold gets larger, fewer bi-asserting clauses are learned and the performance deteriorates. Note that, when $M=10$, the performance of the solver is quite similar to that of Rsat with no bi-asserting clause learning in terms of the number of solved problems.

It is also interesting to note here that the percentages of bi-asserting clauses learned by Rsat+ $(M=1)$ range from $5-15 \%$ on most problems. These percentages roughly reflect the fractions of conflicts for which our learning algorithm was able to find "more useful" learned clauses. Even though bi-asserting clauses account for a relatively small percentage of all learned clauses, they were already able to make significant impact on performance, especially on unsatisfiable problems.

\subsection{Related Work}

In [33], the authors studied various learning schemes (asserting and non-asserting) from a graph-partitioning point of view and compared their performance empirically. Their experimental results showed that the first UIP asserting clause learning scheme was the most robust among the considered schemes.

Non-asserting learning schemes have also been proposed by [29], [17], and [9]. In these papers, non-asserting clauses are learned in addition to asserting clauses (i.e., the solvers may learn multiple clauses for some conflicts). Moreover, additional clauses 


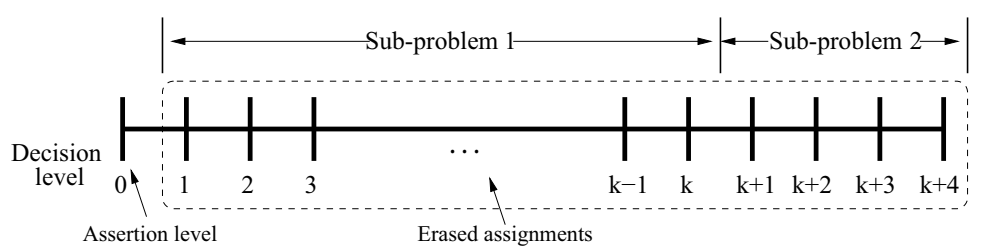

Fig. 3 Erased assignments due to a backtrack in modern SAT solvers.

learned may not be empowering nor bi-asserting. In [29], no specific strategy for learning was mentioned and no experimental results were provided for the proposed learning scheme. In [17], an additional clause is learned opportunistically to bridge the gap between the conflicting clause (the empty clause) and the first UIP assserting clause. The proposed scheme was shown to be effective on hardware and software verification benchmarks. In [9], the learning scheme called local clause recording was proposed. The study was performed on a solver with clause-based decision heuristic [8]. The experimental result presented showed that their learning technique only allowed the solver to solve 4 additional problems.

\section{Avoiding Repeated Work}

In this section, we switch our attention to the other key component of modern SAT solvers-the decision-making engine. We point out one of its inefficiencies, which may cause the solvers to repeatedly undo the progress they make, and propose an effective way for dealing with this problem.

\subsection{An Inefficiency of Existing Decision Making Engines}

As described in Section 2.1, the decision-making engine keeps making progress towards a solution by repeatedly making decisions and deducing implications with unit resolution. However, if a conflict is found, control will be handed over to the clause-learning engine and the decision-making engine will get to resume only after the clause-learning engine has rolled back some decisions and added a learned clause to the knowledge base. Unfortunately, this mechanism posts a potential problem.

According to this scheme, the set of assignments erased by the clause-learning engine may contain many assertions that are unrelated to the current conflict. When this happens, the solver is at risk of losing valuable progress on other parts of the problem. In an extreme case, some of the erased assignments may contain solutions to other independent sub-problems. If the clause-learning engine simply erases these assignments without keeping track of any information, the solver will have to solve these sub-problems again. Figure 3 depicts this situation with two independent subproblems. In this example, the solver has already finished solving sub-problem 1 at level $k$ when a conflict in sub-problem 2 arises at level $k+4$. In this case, the assertion level is 0 (i.e., a unit asserting clause is learned), forcing the solver to erase all assignments including the solution to sub-problem 1 .

To concretely demonstrate the extent of this problem, we performed an experiment on SAT problems with independent component structure. In the following experiment, 


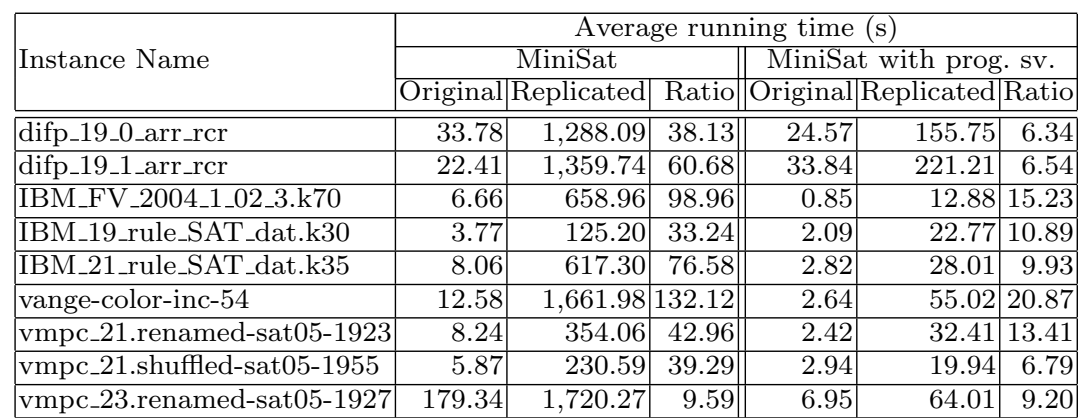

Table 10 Average running time (in seconds) of MiniSat with and without progress saving. The ratio columns show the ratios of average running time on replicated instances over that on original instances.

we artificially generated SAT problems that would cause work repetition in conventional clause-learning SAT solvers. Each problem was generated by concatenating four identical copies of a satisfiable problem. These bigger problems will be referred to as replicated problems throughout this paper.

Table 10 reports the results of this initial experiment, conducted using MiniSat [10], on a computer with a $3.8 \mathrm{GHz}$ processor and $2 \mathrm{~GB}$ of RAM. For each problem, we ran each version of MiniSat 10 times with random initial variable orderings. ${ }^{11}$ The first column of the table shows the name of the problems used and the next 2 columns reports the average running time of MiniSat on each original and replicated problem. The forth column shows the ratio of the average running time on replicated problem to that on the corresponding original problem. As the result shows, solving a replicated problem can be more than two orders of magnitudes less efficient, even though a replicated problem contains four identical copies of the original problem.
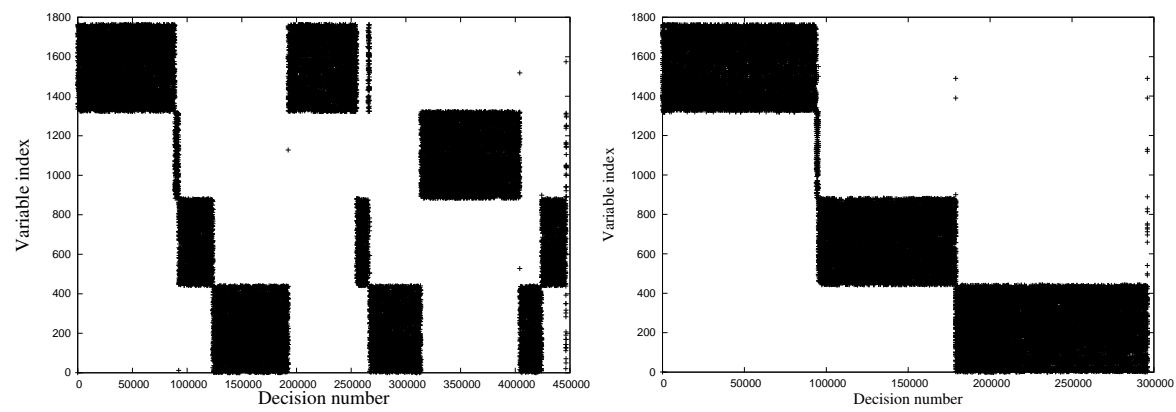

Fig. 4 Decision behavior on a replicated instance of MiniSat (left) and MiniSat with progress saving (right). Both $\mathrm{x}$-axes represent the chronological order of decisions.

Further investigation on these problems reveals the source of inefficiency. In Figure 4 , we plot the indices of decision variables set by MiniSat in chronological order. The

\footnotetext{
11 We set the timeout of each run to 1,800 seconds. Any run with longer running time
} contributed 1,800 seconds in the computation of average running time. 
left plot in Figure 4 shows such a plot based on one run of MiniSat on the replicated instance of vmpc_21.shuffled-sat05-1955. Variable indices in the replicated instance range from 1 to 1764 ( $4 \times 441$ original variables $)$. Each component in the instance occupies a contiguous range of variable indices. Each dark band in this plot indicates the solver's attempt to solve a component. ${ }^{12}$ We can see in the left plot of Figure 4 that MiniSat ended up solving all components multiple times. Most of the attempts to re-solve a component take non-trivial amount of work, as illustrated by the width of each band. This clearly illustrates that work repetition is responsible for a fair amount of the disproportionate increase in runtime of the solver on the replicated instances.

\subsection{Progress Saving}

To deal with the problem of work repetition, in [26], we proposed to deal with this problem with a lightweight component caching technique called progress saving. Progress saving is a simple way of preventing the decision-making engine from having to solve the same sub-problem multiple times. To achieve this, we only need to record the value of every variable assignment that the clause-learning engine erases. This information should then be made available to the decision-making engine. In the future, whenever the decision-making engine decides to make a decision on a variable, it should assign the recorded value to that variable. If the variable chosen for making decision was never assigned a value before, the decision-making engine should proceed with the default heuristic. Note that, according to this algorithm, both decisions and implications should be saved whenever the solvers backtrack. The time and space overhead of this technique is only linear in the number of variables.

In practice, independent sub-problems may exist as parts of the original problem structure or may be created/destroyed dynamically as assignments are made/erased by the solver. A nice property of the proposed technique is that once an independent sub-problem has been solved, each future attempt to solve the sub-problem will require time that is only linear in the size of the sub-problem (without progress saving, this becomes exponential in the worst case). This property holds for the independent subproblem as long as it is not destroyed. ${ }^{13}$

We evaluated the proposed solution on the replicated problems from the previous experiment. The result of this experiment is shown in the last 3 columns of Table 10 . Clearly, progress saving improves the running time of MiniSat by up to an order of magnitude on these problems. The ratios of running time on replicated problems over that on original problems also decrease considerably. In fact, for several of these problems, the ratios are only slightly greater than the number of identical copies (4). Furthermore, the right plot of Figure 4 shows the behavior of MiniSat with progress saving on the replicated problem of vmpc_21.shuffled-sat05-1955. In this case, each sub-problem is "solved" only once (one dark band at any horizontal level). Note that, in the right plot, each sub-problem is still visited more than once as expected. However, each visit after the first attempt on the sub-problem requires little effort from the solver. These later visits correspond to the scattered groups of points after each dark band.

\footnotetext{
12 Our investigation revealed that, in most cases, MiniSat only switched sub-problems when it had finished solving a sub-problem.

13 A sub-problem that is created by variable assignments can be destroyed when some of those assignments are erased.
} 
vmpc_23.renamed-as.sat05-1927

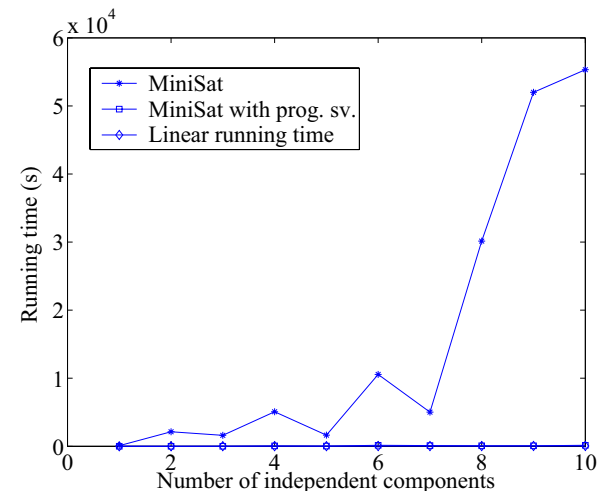

ibm_19_rule_SAT_dat.k30

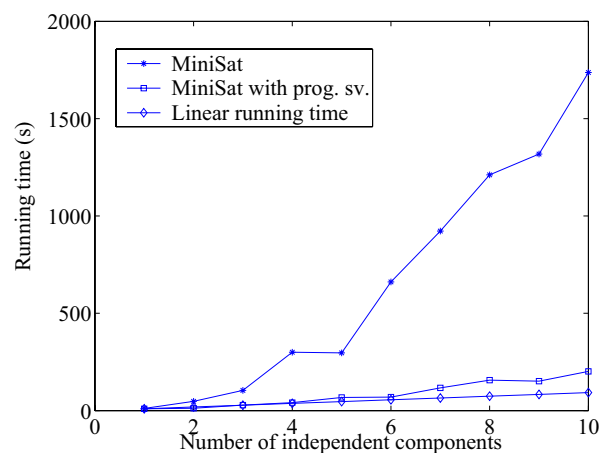

difp_19_0_wal_rcr

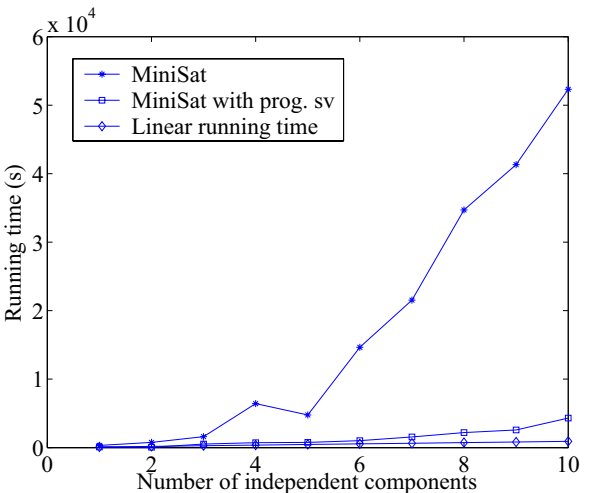

bmc-ibm-10

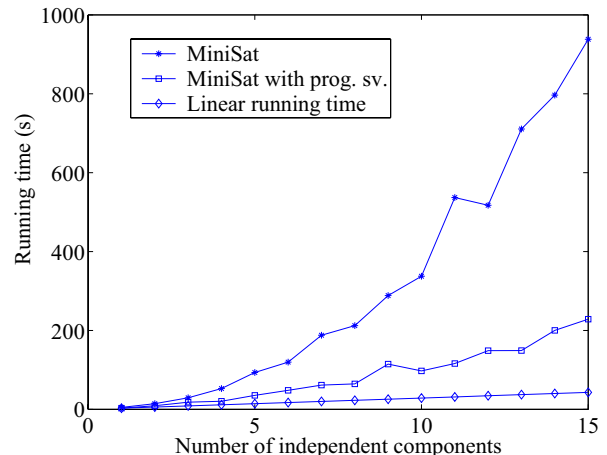

Fig. 5 Running time of MiniSat on replicated instances with varied number of components. Hypothetical linear (on the number of components) running time is also depicted in each plot.

Next, we compare the scalability of MiniSat with and without progress saving. The results are shown in Figure 5. In this experiment, we tested both versions of the solver on problems with varied number of independent sub-problems. The x-axis of each plot in this figure indicates the number of sub-problems presented in the problem, while the $y$-axis corresponds to the running time of the solver. In each plot, we show the running time of both solvers together with the hypothetical linear running time on the problems. According to these plots, the running time of MiniSat without progress saving increases rapidly as the number of sub-problems increases, whereas the running time of MiniSat with progress saving appears to be only slightly worse than linear in the number of sub-problems.

\subsection{Evaluation on Real-World Problems}

We will now present results of evaluating the proposed technique on real-world SAT problems. Most of these problems do not decompose into multiple sub-problems initially. However, after setting some variables, some problems may eventually decompose. 
In [6], the authors demonstrated the prevalence of independent sub-problem structure in real-world instances.

We used 1,403 industrial problems from the SAT competitions and from contemporary benchmark libraries. ${ }^{14}$ All experiments were performed on a machine with a $3.8 \mathrm{GHz}$ CPU and 2GB of RAM, with a time-out limit of 1800 seconds. We considered several versions of MiniSat in this experiment. By default, MiniSat 1.14 always sets the decision variables to false. To demonstrate the effectiveness of progress saving, we also considered two natural modifications to MiniSat's decision-making engine; setting the decision variables to true and setting them to values drawn randomly at decision time.
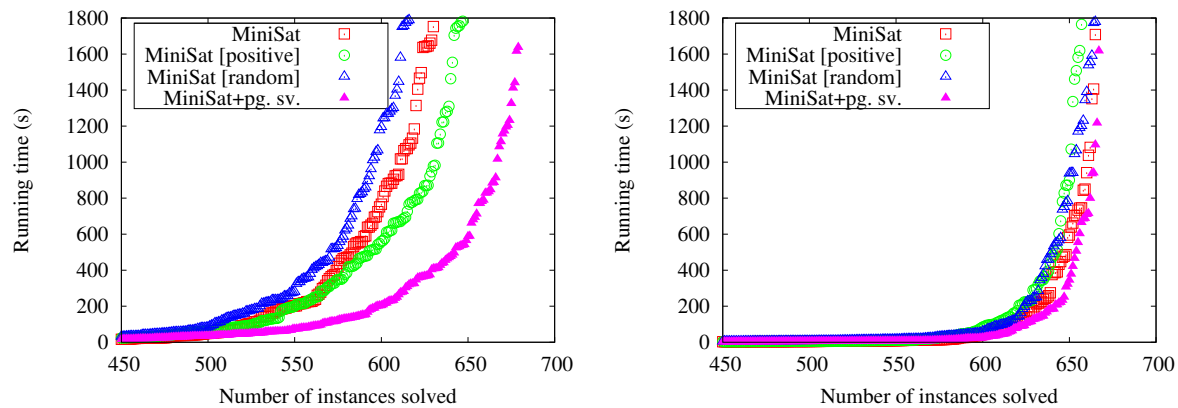

Fig. 6 Running time profiles of three variations of MiniSat and of MiniSat with progress saving. The left plot shows profiles on satisfiable problems while the right plot shows those on unsatisfiable problems.

Figure 6 shows the running time profiles of the considered versions of MiniSat on satisfiable and unsatisfiable problems. First of all, these plots show that simply changing the phase selection heuristic to always set variables positively or randomly does not significantly effect performance either on satisfiable or unsatisfiable problems. However, it is clear that MiniSat with progress saving stands out from other variations on satisfiable problems. It solved 49,32 , and 63 more satisfiable problems than MiniSat, MiniSat [positive], and MiniSat [random], respectively. The right plot of Figure 6 shows that all versions of MiniSat considered essentially have the same performance on unsatisfiable problems. This result demonstrates that even in the cases where there are no solutions to be saved, progress saving does not impair the performance of the solver.

Progress saving was first used in the context of SAT in Rsat [25] in SAT-Race 2006. ${ }^{15}$ Since then, it has been adopted by many other top SAT solvers including MiniSat [30], PicoSat [5], TiniSat [16], and ManySat [32].

\footnotetext{
14 http://www.satcompetition.org, http://www.research.ibm.com/haifa/projects/verification/RB_Homepage/fvbenchmarks.html, http://miroslav-velev.com/sat_benchmarks.html.

15 For more information, see the SAT-Race 2006 website at http://fmv.jku.at/sat-race-2006/.
} 


\subsection{Related Work}

The problem of non-chronological backtracking erasing progress made by the decisionmaking engine has long been observed in the context of CSP. In [13], the author proposed dynamic backtracking as a solution to this problem. This technique allows the solver to specifically undo a bad assignment instead of backtracking to it. This approach is superficially similar to ours. However, it may cause the problem to become overlyconstrained after backtracking, as pointed out by the author. Moreover, implementing this approach in the contemporary SAT framework would require a careful modification to make it work as intended. Neither is the case for our proposed technique.

Frost and Dechter have also experimented with this idea in CSP. In [12], a heuristic called sticking value was used in a CSP solver without any learning and was evaluated only on randomly generated CSP problems. Their experimental results showed that, in the considered settings, this heuristic reduced the running time by a factor of two on a few problems with small-sized variable domains.

In [6], Biere and Sinz showed that independent components do exist in some realworld SAT instances and proposed a method to take advantage of the structure. However, their approach is semi-dynamic, as it only considers permanent decompositions that occur in the absence of any decision and requires a component detection algorithm, which could incur a high overhead. Although experimental results on artificially generated instances shows improvements, no gain was reported on industrial benchmarks.

\section{Conclusions}

We presented a simple model of modern SAT solvers that highlights the roles of the two main components in these solvers: the decision-making engine and the clauselearning engine. Then, based on our model, we discussed two limitations of modern SAT solvers and proposed two techniques for dealing with them. One technique is a new clause-learning scheme that allows the solvers to consider a broader set of learned clauses while maintaining empowerment. The other is a phase selection heuristic that serves as a partial caching mechanism, which reduces a negative effect of the clauselearning engine on the decision-making engine of modern SAT solvers. We demonstrated through experimental results that both proposed techniques significantly improved the performance of the considered solvers.

\section{A Proofs}

This section is entirely dedicated to formalizing and proving the claim made in Section 3.3. The claim itself is formalized in Proposition 1. This proposition is then proved through a series of lemmas (Lemmas 1,2,3).

Before we can state the proposition, we first formalize the type of resolution performed by Algorithm 2.

Definition 1 A resolution derivation of the clause $C_{k}$ from the CNF $\Delta$ is a sequence of clauses $\Pi=C_{1}, C_{2}, \ldots, C_{k}$ where each clause $C_{i}$ is either in $\Delta$ or is a resolvent of clauses preceding $C_{i}$. Furthermore,

- $\Pi$ is linear if each clause $C_{i}$ for $i \geq 3$ is either in $\Delta$ or is the resolvent of $C_{i-2}$ and $C_{i-1}$. The clauses $C_{1}, C_{2}, C_{4}, C_{6}, C_{8}, \ldots$ of a linear resolution are called the non-resolvents of the derivation. 
- $\Pi$ is causal if it is linear and if the resolved variable $x$ of $C_{i}$ and $C_{i+1}$ does not appear in clauses $C_{i+2}, \ldots, C_{k}$.

We now formalize the claim using the above definition.

Proposition 1 Let $\Pi$ be a causal resolution derivation of the clause $C$. $C$ is empowering with respect to the non-resolvents in $\Pi$ if $\Pi$ contains a merge resolution step.

We will prove this proposition with a series of lemmas. The proof makes extensive use of the fact that for a conjunction of literals $\sigma$ and CNF $\Delta$, we have $\Delta \wedge \sigma=(\Delta \mid \sigma) \wedge \sigma$. Here, $\Delta \mid \sigma$ is the CNF obtained by removing any clause in $\Delta$ that mentions any literal in $\sigma$ and removing any literal from $\Delta$ whose negation appears in $\sigma$. Hence, none of the variables in $\sigma$ will appear in $\Delta \mid \sigma$. The following formal definition of empowerment is needed in the proofs.

Definition 2 (Empowerment [28]) Let $\alpha \Rightarrow \ell$ be a clause where $\ell$ is a literal and $\alpha$ is a conjunction of literals. The clause is empowering with respect to CNF $\Delta$ via $\ell$ iff

1. $\Delta \models(\alpha \Rightarrow \ell)$ : the clause is implied by $\Delta$.

2. $\Delta \wedge \alpha \forall \ell$ : the literal $\ell$ cannot be derived from $\Delta \wedge \alpha$ using unit resolution.

The following lemma gives the basis for generating empowering clauses.

Lemma 1 (Generation of Empowerment) If $C_{3}$ is the resolvent of a merge resolution between $C_{1}=(x \vee \ell \vee \alpha)$ and $C_{2}=(\neg x \vee \ell \vee \beta)$, then $C_{3}$ is empowering with respect to $C_{1} \wedge C_{2}$ and $\ell$ is its empowering literal.

Proof Let $\sigma=\neg(\alpha \vee \beta)$. Clearly, $C_{3}=\sigma \Rightarrow \ell$. Consider $C_{1} \wedge C_{2} \wedge \sigma=\left(C_{1} \wedge C_{2}\right) \mid \sigma \wedge \sigma=$ $(\ell \vee x) \wedge(\ell \vee \neg x) \wedge \sigma$. Clearly, unit resolution cannot derive $\ell$ from this. Hence, $C_{3}$ is empowering with respect to $C_{1} \wedge C_{2}$ and $\ell$ is an empowering literal.

Lemma 2 (Backward Preservation of Empowerment) Let $C_{1}, C_{2}, \ldots, C_{k}$ be a causal resolution. If $C_{k}$ is empowering with respect to $\left\{C_{3}, \ldots, C_{k-1}\right\}$, then $C_{k}$ is also empowering with respect to $\left\{C_{1}, \ldots, C_{k-1}\right\}$.

This lemma holds because $C_{3}$, the resolvent of $C_{1}, C_{2}$, is essentially $\exists x\left(C_{1} \wedge C_{2}\right)$, where $x$ is the resolved variable. Hence, adding $C_{1}, C_{2}$ to the formula that already contains $C_{3}$ cannot give us any new knowledge other than that on the resolved variable, which does not appear elsewhere in the derivation (because it is causal). Therefore, $C_{k}$ must still be empowering with respect to the new knowledge base.

The combination of the above lemmas show that the final product of a causal resolution derivation whose last step is a merge resolution is always empowering with respect to the non-resolvents of the derivation. The next lemma shows that once an empowering clause is obtained from a causal derivation, all further derived clauses will also be empowering.

If $\Pi=C_{1}, \ldots, C_{n}$ is a linear resolution derivation, we will use $N R(\Pi)$ to denote the non-resolvents of $\Pi$ (i.e., $N R(\Pi)=\left\{C_{1}\right\} \cup\left\{C_{i} \mid 2 \leq i \leq n, i\right.$ is even $\}$ ).

Lemma 3 (Forward Preservation of Empowerment) Let $\Pi^{\star}=C_{1}, \ldots, C_{n}$ and $\Pi=$ $\Pi^{\star}, C_{n+1}, C_{n+2}$, where $C_{n+2}=C$, be causal resolution derivations from clauses in $\Delta$. If $C_{n}$ is empowering with respect to $N R\left(\Pi^{\star}\right)$, then $C$ is empowering with respect to $N R(\Pi)$.

Proof Clearly, $N R(\Pi)=N R\left(\Pi^{\star}\right) \wedge C_{n+1}$. Let $x$ be the resolved variable of $C_{n}$ and $C_{n+1}$. We may assume that $C_{n}$ and $C_{n+1}$ do not share a literal. ${ }^{16}$ With this assumption, we have that

$$
C_{n+1} \text { and the clauses of } N R\left(\Pi^{\star}\right) \text { cannot share any variable other than } x \text {. }
$$

Otherwise, any common variable between $C_{n+1}$ and $N R\left(\Pi^{\star}\right)$ must remain unresolved in $\Pi^{\star}$ (because $\Pi$ is causal, which does not allow any resolved variable to reappear) and must therefore appear in $C_{n}$, which would contradiction our assumption that $C_{n}$ and $C_{n+1}$ share no common literal. Let $\ell$ be the empowering literal of $C_{n}$ (with respect to $N R\left(\Pi^{\star}\right)$ ). Because $C_{n}$ is empowering, we have

$$
N R\left(\Pi^{\star}\right) \wedge \neg\left(C_{n} \backslash\{\ell\}\right) \forall \ell .
$$

There are two cases to consider:

16 Otherwise, $C$, is empowering with respect to $N R(\Pi)$ by the results in Lemmas 1,2 . 
1. $\ell \neq x$. Assume, WLOG, that $C_{n}=(x \vee \ell \vee \alpha)$ and $C_{n+1}=(\neg x \vee \beta)$. We then have $C=(\neg \alpha \wedge \neg \beta) \Rightarrow \ell$. Consider $N R(\Pi) \wedge \neg \alpha \wedge \neg \beta=N R\left(\Pi^{\star}\right) \wedge C_{n+1} \wedge \neg \alpha \wedge \neg \beta$. In this case, $C_{n+1}$ can only generate $\neg x$ as an implication. However, this will not allow $N R\left(\Pi^{\star}\right)$ to produce $\ell$, because we know from (2) that $N R\left(\Pi^{\star}\right) \wedge \neg \alpha \wedge \neg x \forall \ell$. Since, from (1), $N R\left(\Pi^{\star}\right)$ does not mention any variable in $\beta$, we can safely conclude that $N R(\Pi) \wedge \neg \alpha \wedge \neg \beta \nvdash \ell$.

2. $\ell=x$ or $\ell=\neg x$. Assume, WLOG, $C_{n}=\ell \vee \alpha$ and $C_{n+1}=\neg \ell \vee \beta$. Let $y$ be any literal in $\beta$ and $\sigma=\neg(C \backslash\{y\})$. Clearly, $C=\sigma \Rightarrow y$. Now, consider $N R(\Pi) \wedge \sigma=N R\left(\Pi^{\star}\right) \wedge C_{n+1} \wedge \sigma$. The literal $y$ only appears in $C_{n+1}$ which becomes $(\neg \ell \vee y)$ under $\sigma$. However, $N R\left(\Pi^{\star}\right) \wedge \sigma$ cannot produce $\ell$, which is needed by $C_{n+1}$ to imply $y$, because we know that, from (1), $N R\left(\Pi^{\star}\right)$ does not mention any variable in $\beta$, and that, from (2), $N R\left(\Pi^{\star}\right) \wedge \neg \alpha \forall \ell$. Therefore, $N R(\Pi) \wedge \sigma \nvdash \ell$.

Hence, $C$ is empowering wrt $N R(\Pi)$ in both cases.

Together, these lemmas prove the result in Proposition 1.

\section{References}

1. Andrews, P. B. Resolution with merging. J. ACM 15, 3 (1968), 367-381.

2. Audemard, G., Bordeaux, L., Hamadi, Y., Jabbour, S., and Sais, L. A generalized framework for conflict analysis. In SAT (2008), pp. 21-27.

3. Bacchus, F., AND WinTER, J. Effective preprocessing with hyper-resolution and equality reduction. In In SAT (2003), pp. 341-355.

4. Beame, P., Kautz, H., and Sabharwal, A. Towards understanding and harnessing the potential of clause learning. JAIR 22 (2004), 319-351.

5. Biere, A. Picosat essentials. Journal on Satisfiability, Boolean Modeling and Computation (JSAT) (2008), 75-97.

6. Biere, A., And Sinz, C. Decomposing sat problems into connected components. Journal on Satisfiability, Boolean Modeling and Computation (JSAT) 2 (2006).

7. Davis, M., Logemann, G., And Loveland, D. A machine program for theorem-proving. Commun. ACM 5, 7 (1962), 394-397.

8. Dershowitz, N., Hanna, Z., And Nadel, A. A clause-based heuristic for sat solvers. In Proceedings of 8th International Conference on Theory and Applications of Satisfiability Testing(SAT) (2005), pp. 46-60.

9. Dershowitz, N., Hanna, Z., And Nadel, A. Towards a better understanding of the functionality of a conflict-driven sat solver. In $S A T$ (2007), pp. 287-293.

10. EÉn, N., AND Sörensson, N. An extensible sat-solver. In SAT (2003), pp. 502-518.

11. En, N., AND BiERE, A. Effective preprocessing in sat through variable and clause elimination. In In proc. SAT05, volume 3569 of LNCS (2005), Springer, pp. 61-75.

12. Frost, D., AND Dechter, R. In search of the best constraint satisfaction search. In AAAI '94: Proceedings of the twelfth national conference on Artificial intelligence (vol. 1) (Menlo Park, CA, USA, 1994), American Association for Artificial Intelligence, pp. 301-306.

13. Ginsberg, M. L. Dynamic backtracking. Journal of Artificial Intelligence Research 1 (1993), 25-46.

14. GoldBerg, E., AND Novikov, Y. Verification of proofs of unsatisfiability for cnf formulas. In Proceedings of DATE2003 (2003).

15. Hertel, P., Bacchus, F., Pitassi, T., and Van Gelder, A. Clause learning can effectively p-simulate general propositional resolution. In Proc. of AAAI-08 (2008), pp. 283290.

16. Huang, J. A case for simple sat solvers. In CP-07 (2007), pp. 839-846.

17. Jin, H., AND Somenzi, F. Strong conflict analysis for propositional satisfiability. In DATE '06: Proceedings of the conference on Design, automation and test in Europe (3001 Leuven, Belgium, Belgium, 2006), European Design and Automation Association, pp. 818-823.

18. Le Berre, D. Exploiting the real power of unit propagation lookahead. In Workshop on Theory and Applications of Satisfiability Testing(SAT'01) (Boston University, Massachusetts, USA, June 2001), H. Kautz and B. Selman, Eds., Elsevier Science Publishers, pp. $59-80$.

19. LI, C. M. Heuristics based on unit propagation for satisfiability problems. In Proceedings of IJCAI-97 (1997), pp. 366-371. 
20. LI, C. M. A constraint-based approach to narrow search trees for satisfiability. Inf. Process. Lett. 71, 2 (1999), 75-80.

21. Lynce, I., And Marques-Silva, Jo A. Probing-based preprocessing techniques for propositional satisfiability. In ICTAI '03: Proceedings of the 15th IEEE International Conference on Tools with Artificial Intelligence (Washington, DC, USA, 2003), IEEE Computer Society, p. 105.

22. Marques-Silva, J. P., and Sakallah, K. A. GRASP - A New Search Algorithm for Satisfiability. In Proceedings of IEEE/ACM International Conference on Computer-Aided Design (1996), pp. 220-227.

23. Moskewicz, M., Madigan, C., Zhao, Y., Zhang, L., and Malik, S. Chaff: Engineering an efficient sat solver. pp. 530-535.

24. Nieuwenhuis, R., Oliveras, A., and Tinelli, C. Solving sat and sat modulo theories: From an abstract davis-putnam-logemann-loveland procedure to DPLL(T). Journal of ACM 53, 6 (2006), 937-977.

25. Pipatsrisawat, K., And Darwiche, A. Rsat 1.03: Sat solver description. Tech. Rep. D-152, Automated Reasoning Group, Computer Science Department, UCLA, 2006.

26. Pipatsrisawat, K., AND Darwiche, A. A lightweight component caching scheme for satisfiability solvers. In Proceedings of 10th International Conference on Theory and Applications of Satisfiability Testing(SAT) (2007), pp. 294-299.

27. Pipatsrisawat, K., And Darwiche, A. Rsat 2.0: Sat solver description. Tech. Rep. D-153, Automated Reasoning Group, Comp. Sci. Department, UCLA, 2007.

28. Pipatsrisawat, K., And Darwiche, A. A new clause learning scheme for efficient unsatisfiability proofs. In Proceedings of the Twenty-Third AAAI Conference on Artificial Intelligence (AAAI) (2008), pp. 1481-1484.

29. RyAn, L. Efficient Algorithms for Clause-Learning SAT Solvers. Master's thesis, Simon Fraser University, 2004

30. Sörensson, N., And É́n, N. Minisat 2.1 and minisat++ 1.0-sat race 2008 editions, 2008.

31. Subbarayan, S., , Subbarayan, S., and Pradhan, D. K. Niver: Non increasing variable elimination resolution for preprocessing sat instances. In In Proc. 7th International Conference on Theory and Applications of Satisfiability Testing (SAT (2004), Springer, pp. 276-291.

32. Youssef Hamadi, S. J., And SaIs, L. Manysat: solver description. Tech. Rep. MSR-TR2008-83, 2008

33. Zhang, L., Madigan, C. F., Moskewicz, M. W., and Malik, S. Efficient conflict driven learning in boolean satisfiability solver. In $I C C A D$ (2001), pp. 279-285. 